\title{
Review of: "Smoking, vaping and hospitalization for COVID-19"
}

\author{
Jason Sheltzer \\ 1 Cold Spring Harbor Laboratory
}

This preprint contrasts two statistics: according to a survey conducted by the WHO, $\sim 27 \%$ of people in China are smokers. If you look at coronavirus hospital admissions in China, fewer than $27 \%$ are smokers. Ergo, the paper suggests, smoking may protect against COVID19.

This comparison assumes that the definition of smoker is constant. But, that assumption is wrong. One paper included in this analysis (Chen et al.) actually uses a cutoff of 30 pack-years (219,000 cigarettes!) to identify smokers. They aren't recording all smokers, just heavy smokers. This 30 pack-year threshold was not used in the WHO national survey that produced the $27 \%$ estimate, so it's absolutely wrong to directly compare these two values as if they're measuring the same thing.

In order to further investig ate, I emailed the authors of some of the studies on COVID-19 in China. One doctor (on this manuscript: https://papers.ssrn.com/sol3/papers.cfm? abstract_id=3543584) replied that their smoking frequency was so low because some patients were literally too sick to ask: "In our paper on COVID-19 characteristics, many patients cannot answer questions about whether to smoke because of their older age and serious illness. Therefore, the proportion of smoking in patients with COVID-19 is low."

These two examples highlight the first problem with this preprint: it's clear that in these studies, the definition of a smoker is neither consistent nor consistently applied. You can't throw them all on a graph and call it a meta-analysis.

The second problem is that it's absolutely wrong to assume that " $27 \%$ " is an appropriate base rate for comparisons. To see this, take a look at Table 3 of Guan et al. (Eur Respir J.) from this preprint. They report the frequency of various comorbidities among Chinese patients, broken out between ever-smokers and never-smokers. In their analysis, only $13 \%$ of COPD patients, $8 \%$ of hypertensives, and $10 \%$ of people with cardiovascular 
disease are ever-smokers. Assuming a base rate of $27 \%$ smokers in China, this analysis would falsely suggest that smoking protects against lung and heart disease!

The best approach would be to look at the frequency of smoking in a defined cohort of patients who are tested and found to be either positive or negative for COVID-19. That way you can apply a consistent definition of "smoker" and get an accurate base frequency. So far, I can find one published example that directly compares COVIDpositive and COVID-neg ative patients:

https://onlinelibrary.wiley.com/doi/full/10.1002/jmv.25763. In this study (not cited by the preprint), 19\% of COVID-positive patients were smokers and 15\% of COVID-negative patients were smokers. This data argues that smoking does not actually protect ag ainst coronavirus infections. Additionally, it shows that when hospitals are directly recording smoking statuses, they observe the prevalence of smoking to be below $27 \%$.

Similarly, if you compare patients with critical and mild COVID-19, multiple studies indicate that smoking is strongly associated with severe infections. In one recent cohort, $62 \%$ of severe cases were smokers, compared to only $10 \%$ of mild cases: https://www.medrxiv.org/content/10.1101/2020.03.01.20029397v1.full.pdf.

So, I think that the analysis in this preprint is severely flawed. They're comparing statistics that shouldn't be compared. The Chen et al. study should be removed and the Zhu et al. study should be added and addressed. In short, I don't think that there's any convincing evidence to suggest that smoking protects against coronavirus. 